\title{
ESTUDO COMPARATIVO DE MÉTOdOS PARA A AVALIAÇÃO DO VIGOR DE SEMENTES DE SORGO (Sorghum vulgare PERS.)*
}

\author{
FRANCISCO H. DUBbERn DE SOUZA** \\ JULIO MARCOS FILHO***
}

\begin{abstract}
RESUMO
O presente trabalho foi conduzido no sentido de comparar a eficiência de diferentes métodos para avaliar a qualidade de sementes de sorgo (Sorghum vulgare Pers.).

Foram comparados entre si os seguintes métodos: teste padrão de germinação, primeira contagem de germinação, velocidade de germinação, teste do cloreto de amónio, envelhecimento rápido, velocidade e porcentagem de emergência no campo.

As análises dos dados e a interpretação dos resultados permitiram concluir que a eficiência dos métodos estudados depende da qualidade das sementes.

O teste do cloreto de amônio e a velocidade de germinação foram os mais sensíveis às diferenças de vigor entre os tratamentos, enquanto que o teste padrão e a primeira contagem de germinação revelaram apenas as diferenças marcantes de qualidade.
\end{abstract}

\section{INTRODUÇÃO}

O processo de deterioração de sementes, segundo DELOUCHE \& BASKIN (1973), é pouco conhecido, mas algumas de suas conseqüências têm sido observadas por diferentes pesquisadores; dentre elas destacam-se a maior sensibilidade a condições ambientais adversas, a diminuição da velocidade de germinação e do crescimento das plantas, o aumento da ocorrência de anormalidades em plântulas e, como conseqüência final, a perda da capacidade de germinação.

Portanto, as sementes podem germinar mesmo em um estágio avançado de deterioração. Desta forma, fica evidenciada a deficiência do teste padrão de germinação que, sendo conduzido sob condições artificiais muito favoráveis, não traduz as possibilidades das sementes resistirem a condições adversas.

As recentes pesquisas têm conferido importância cada vez maior ao vigor como fator de qualidade. A literatura sobre o assunto revela que muitos métodos foram testados e indicados para diferentes espécies, mas o sorgo (Sorghum vulgare Pers.), cujas sementes são muito sensiveis à deterioração e apresentam sérios problemas de qualidade, raramente faz parte desses trabalhos (VANDERLIP e outros, 1973); no Brasil, praticamente não foi estudado até o momento.

* Entregue para publicação em 27/11/1975.

** Engọ Agrọ - Convênio MA/AGIPLAN/USP.

*** Prof. Assist. Dr. - Departamento de Agricultura e Horticultura, ESALQ, USP. 
Assim, o presente trabalho foi planejado e conduzido com o objetivo de comparar a eficiência de diferentes testes para a avaliação do vigor de sementes de sorgo, armazenadas sob duas condições de ambiente.

\section{REVISÃo dA LITERATURA}

SWANSON \& HUNTER (1936) mostraram que a discrepância entre a porcentagem de germinação obtida em laboratório e a de emergência no campo é, em muitos casos, de 30 a 50\%, mesmo quando as sementes são de alta qualidade. Essas diferenças, segundo esses pesquisadores, são maiores com as sementes de sorgo que com as outras espécies devido aos problemas causados pelo clima durante a maturação e colheita e às injúrias mecânicas durante a colheita $\mathrm{e} o$ processamento.

MIRANDA (1967), armazemou sementes de sorgo em câmara seca (45 a 60\% de umidade relativa) e em condiçōes normiais de ambiente. Verificou, após um ano, que as flutuações periódicas nos teores de umidade das sementes, ocorridas devido à conservação em ambiente normal prejudicaram significativamente o poder germinativo, principalmente quando a umidade relativa era superior a $70 \%$.

Trabalhando com sementes de sorgo, HELMER (1967) observou que o teste de envelhecimento precoce foi eficiente para avaliar o vigor, bem como para determinar o potencial de conservação de diferentes lotes de sementes; obteve resultados semelhantes aos de CLARK (1953), DELOUCHE (1963) e GRABE (1965), segundo os quais o teste de germinação não é capaz de revelar a real condição fisiológica de um lote de sementes.

ABDULAHI (1968) testou diferentes métodos para determinar a qualidade de sementes de sorgo: teste padrão de germinação, envelhecimento rápido (380C e $95-$ $100 \%$ de umidade relativa, durante dez dias), teste de frio, cloreto de amônio e emergência no campo. Concluiu que o teste do cloreto de amônio (2\%), a $40 \circ \mathrm{C}$, durante duas horas, foi o que melhor identificou os diferentes níveis de deterioração dos lotes, além de apresentar alta correlação com a porcentagem de emergência no campo.

Trabalhando com diferentes híbridos de sorgo, MOCKEL (1969) comparou os resultados do teste padrão de germinação, primeira contagem, cloreto de amônio, porcentagem de sementes pequenas e tetrazólio, com a porcentagem de emergência das plântulas no campo. Dos métodos testados, o cloreto de amônio foi o mais eficiente na avaliação do vigor e o que mais se correlacionou com a emergência no campo. Os resultados do teste padrão de germinação se correlacionaram com os demais e com a emergência no campo apenas quando as sementes eram de baixa qualidade; a primeira contagem, por sua vez, não revelou os diferentes níveis de vigor entre as amostras testadas. Resultados semelhantes foram obtidos por ABDULAHI \& VANDERLIP (1972) e por VANDERLIP e colaboradores (1973), que também teceram críticas ao teste de germinação.

CAMARGO (1971) utilizou diferentes métodos para determinar a qualidade de sementes de sorgo, concluindo que o indice de velocidade dè germinaçáo foi o que deu origem à melhor correlação com a emergência no campo. 
YAYOCK e outros (1975), baseando-se em pesquisas referentes à eficiência do teste do clore to de amônio, estudaram variações da concentração e do tempo de imersão das sementes na solução desse sal. Concluíram que nenhuma das modificaçðes introduzidas na técnica descrita por ABDULAHI (1968) aumentou significativamente a precisão do teste ou a sua correlação com a porcentagem de emergência das plântulas no campo.

\section{MATERIAIS E MÉTODOS}

1. Sementes - Os testes foram conduzidos com sementes de sorgo híbrido Continental 2201, com 100\% de pureza e 90\% de germinação, obtidas junto à Companhia Continental de Cereais (CONTIBRASIL) em Ribeirão Preto, SP.

Após permanecer durante 45 dias em câmara seca para a uniformização do teor de umidade, o material foi dividido em duas partes iguais; uma foi submetida a um processo de envelhecimento artificial em câmara de envelhecimento precoce a $420 \mathrm{C} \mathrm{e}$ $100 \%$ de umidade relativa, durante 120 horas, enquanto a outra não recebeu esse tratamento.

Posteriormente, preparou-se duas amostras: a primeira, através de uma mistura na proporção de 7:3 entre a parte não envelhecida e a envelhecida, respectivamente, seguida de cuidadosa homogeinização; a segunda, constituída apenas da parte não envelhecida.

Esse procedimentu foi adotado para a obtenção de duas amostras com diferentes níveis de deterioração: um com sementes não envelhecidas (NE) e a outra com sementes envelhecidas (E). Técnica semelhante foi empregada por CAMARGO (1971).

2. Ambientes de Conservação - As amostras de sementes envelhecidas e de não envelhecidas foram divididas em duas porções iguais e conservadas em dois ambientes: em câmara seca, do Laboratório de Sementes do Departamento de Agricultura e Horticultura, ESALQ/USP, com umidade relativa de $35 \%$ e temperatura média de $23{ }^{\circ} \mathrm{C}$ $\left(A_{1}\right)$ e em condições normais de ambiente do referido laboratório $\left(A_{2}\right)$.

3. Periodo de Armazenamento - As sementes foram conservadas nos ambientes citados, de junho de 1974 a junho de 1975. Periodicamente, com intervalos semestrais, avaliou-se os efeitos dos tratamentos através de testes de germinação e de testes de vigor, instalados em junho e dezembro de 1974 e junho de 1975, designados neste trabalho por $\mathrm{P}_{1}, \mathrm{P}_{2}$ e $\mathrm{P}_{3}$, respectivamente.

4. Testes de Germinação - A germinação se processou em um aparelho STULTS, sob temperaturas alternadas de $20-30^{\circ} \mathrm{C}$. Utilizou-se caixas plásticas GER-BOX, onde as sementes foram semeadas sobre papel chupão especial para germinação.

Foram instaladas 6 repetições de 50 sementes por tratamento e as interpretações efetuadas no 50 e 10 o a a partir do início de cada teste, segundo os critérios estabelecidos nas Regras para Análise de Sementes (MINISTÉRIO DA AGRICULTURA, 1967). 


\section{Testes de Vigor}

5.1. Primeira Contagem de Germinação - Os dados da primeira contagem do teste de germinação descritos no item 4 , foram computados, segundo o método utilizado por BYRD \& DELOUCHE (1971).

5.2. Velocidade de Germinação - Foi instalado de maneira idêntica à descrita no item 4, com 6 repetições de 50 sementes para cada um dos tratamentos. Retir̃ou-se diariamente do substrato as plântulas normais com plúmula de comprimento superior a $0,02 \mathrm{~m}$, até que toda a germinação foi completada.

A seguir calculou-se um índice de vigor para cada repetição segundo o método descrito por CAMARGO. (1971).

5.3. Teste do Cloreto de Amônio - Este método utilizado por ABDULAHI (1968) consistiu na embebição de 300 sementes de cada tratamento em uma solução de cloreto de amônio a $2 \%$, durante duas horas, a $40^{\circ} \mathrm{C}$; a seguir, as sementes foram lavadas em água corrente e colocadas para germinar; o teste foi interpretado no 50 dia após a sua instalação.

5.4. Envelhecimento Rápido - Na condução deste teste as sementes de cada um dos tratamentos foram colocadas em uma câmara de envelhecimento precoce, de marca DE LEO, em cujo interior a temperatura era de $42{ }^{\circ} \mathrm{C}$ e a umidade relativa de $100 \%$.

Foram empregados dois períodos de envelhecimento: 5 dias e 7 dias; para cada um deles foram tomadas 300 sementes de cada tratamento. Decorridos esses períodos, as sementes eram retiradas da câmara e colocadas para germinar. As contagens foram feitas ao término de cinco dias de permanência no germinador.

5.5. Emergência das Plântulas - Estes testes foram conduzidos em canteiros da Casa-de-Vegetação do Departamento de Agricultura e Horticultura, ESALQ/USP.

Foi adotado esquema inteiramente casualizado, com seis repetições de cada tratamento, num total de 24 parcelas. Cada parcela constava de uma linha de $1,0 \mathrm{~m}$ onde se distribuíam 30 sementes; uma linha distava $0,20 \mathrm{~m}$ da outra. As parcelas eram irrigadas a cada dois dias.

Em cada parcela foram obtidos dados de velocidade de emergência, através da contagem diária das plântulas cuja plúmula apresentava comprimento superior a $0,02 \mathrm{~m}$; esses dados foram posteriormente transformados em índices de vigor, segundo o método descrito em CAMARGO (1971).

Quando a emergência se completou, foi determinada a porcentagem de emergência das plântulas. 
6. Procedimento Estatístico - Os dados obtidos nos testes de germinação e de vigor foram submetidos à análise da variância segundo esquema fatorial encontrado em seguida:

\begin{tabular}{lc}
\hline Causas de Variação & Graus de Liberdade \\
\hline \hline Envelhecimento (E) & 1 \\
Ambientes (A) & 1 \\
Períodos de Armazenamento (P) & 2 \\
E X A & 1 \\
E X P & 2 \\
A X P & 2 \\
E X A X P & 2 \\
Resíduo & 60 \\
\hline Total & 71 \\
\hline
\end{tabular}

Para tanto, os dados de germinaçâo, primeira contagem, cloreto de amônio, envelhecimento rápido e porcentagem de emergência foram transformados em arc sen $\sqrt{\%}$ e os de velocidade de germinação e velocidade de emergência, em $\sqrt{x}$. As médias foram comparadas pelo método de Tukey.

Posteriormente, coeficientes de correlação simples (r) foram calculados para todas as combinações entre os testes de germinação e de vigor, para os tratamentos $\mathrm{NE} \mathrm{A}_{1}$, $\mathrm{E} \mathrm{A}_{1}, \mathrm{NE} \mathrm{A}_{2}$ e $\mathrm{E} \mathrm{A}_{2}$. teste $\mathrm{t}$.

A significância dos coeficientes de correlação simples foram determinados pelo

\section{RESULTADOS E DISCUSSÃO}

O estudo da eficiência de diferentes métodos para a avaliação do vigor de sementes de sorgo e das correlações entre os mesmos foi o objetivo do presente trabaiho.

As análises da variância dos dados obtidos revelaram que os métodos testados acusaram diferenças entre os efeitos dos tratamentos, conforme se observa através do exame do quadro 1.

Os efeitos de envelhecimento, de ambientes e de períodos de armazenamento foram significativos ao nível de $1 \%$ de probabilidade tanto para os testes de gemminação como para os de vigor. Porém esses tratamentos apresentaram efeitos interdependentes; como se pode constatar pelo quadro 1 , houve significância para interações. 


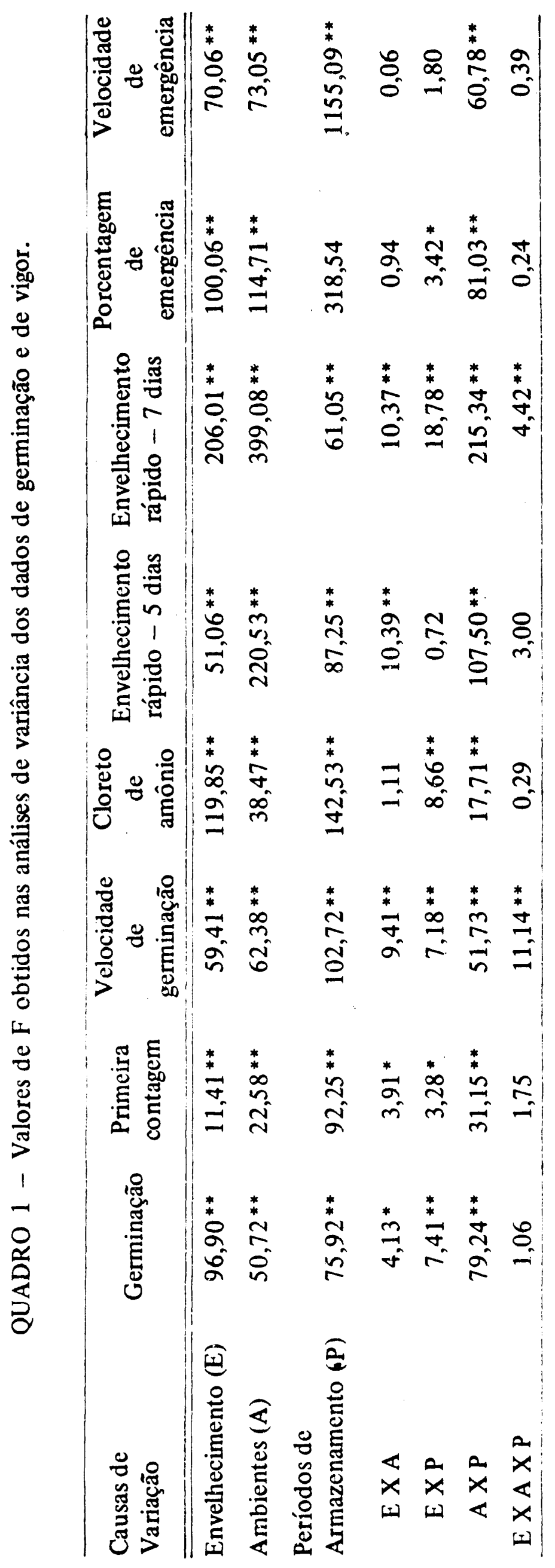


1. Interação Envelhecimento $\mathbf{X}$ Ambientes - Os valores médios obtidos para a interação Envelhecimento $X$ Ambientes, referentes aos testes de germinação e de envelhecimento rápido ( 5 dias) encontram-se no quadro 2.

QUADRO 2 - Médias obtidas para a interação Envelhecimentos X Ambientes.

\begin{tabular}{cccc}
\hline & Germinação & & \multicolumn{2}{c}{ Envelhecimento rápido - 5 dias } \\
\hline Tratamentos & Médias & Tratamentos & Médias \\
\hline NE A $_{1}$ & 62,18 & $\mathrm{NE} \mathrm{A}_{1}$ & 42,88 \\
$\mathrm{NE} \mathrm{A}_{2}$ & 57,79 & $\mathrm{E} \mathrm{A}_{1}$ & 34,52 \\
$\mathrm{E} \mathrm{A}_{1}$ & 55,44 & $\mathrm{NE} \mathrm{A}_{2}$ & 28,31 \\
$\mathrm{E} \mathrm{A}_{2}$ & 47,54 & $\mathrm{E} \mathrm{A}_{2}$ & 25,15 \\
\hline d.m.s. $-5 \%$ & 3,23 & & 3,71 \\
(Tukey) & & & 10,46 \\
\hline C.V.\% & 6,57 & & \\
\hline
\end{tabular}

Houve efeito benéfico do ambiente controlado $\left(\mathrm{A}_{1}\right)$ sobre a germinação e vigor, tanto para o tratamento $\mathrm{E}$ como para o NE, observando-se também um efeito significativo desfavorável do tratamento $\mathrm{E}$ sobre a germinação.

Nos testes de envelhecimento rápido ( 5 dias) o efeito desfavorável do tratamento $\mathrm{E}$ não se manifestou quando as sementes foram armazenadas em ambiente normal $\left(\mathrm{A}_{2}\right)$. Observou-se ainda, que as sementes correspondentes ao tratamento $\mathrm{E}$, armazenadas em câmara seca $\left(E A_{1}\right)$ foram mais vigorosas que as do tratamento $\mathrm{NE}$ armazenadas em ambiente normal $\left(\mathrm{NE} \mathrm{A}_{2}\right)$; esta ocorrência não se verificou quanto à germinação, onde as rnédias destes tratamentos foram estatisticamente semelhantes.

Estes resultados são semelhantes aos observados por HELMER (1967) quanto à eficiência do teste de envelhecimento rápido para avaliar o vigor de sementes de sorgo, embora este pesquisador tenha utilizado um período diferente de permanência das sementes na câmara daqueles utilizados no presente trabalho. Por outro lado, estão de acordo com as críticas emitidas por DELOUCHE (1963), MOCKEL (1969) e CAMARGO (1971), que constataram deficiências do teste padrão de germinação para distinguir pequenas diferenças entre a qualidade de amostras de sementes.

2. Interação Envelhecimentos X Períodos de Armazenamento - As médias dos valores observados para esta interação, nos diferentes testes, acham-se no quadro 3.

Os resultados de germinação, de porcentagem de emergência e de cloreto de amônio acusaram efeitos negativos do envelhecimento nas três primeiras épocas estudadas, ou seja, em $\mathrm{P}_{1}, \mathrm{P}_{2}$ e $\mathrm{P}_{3}$ as médias obtidas para o tratamento $\mathrm{E}$ foram inferiores às das sementes correspondentes a NE. Por outro lado, os resultados da primeira contagem de germinação acusaram efeitos negativos do tratamento $E$ apenas na terceira época $\left(\mathrm{P}_{3}\right)$. 
QUADRO 3 - Médias obtidas para a ineração Envelhecimentos X Períodos de Armazenamento.

\begin{tabular}{|c|c|c|c|c|c|c|c|}
\hline \multicolumn{2}{|c|}{ Germinação } & \multicolumn{2}{|c|}{ Primeira contagem } & \multicolumn{2}{|c|}{ Cloreto de amônio } & \multicolumn{2}{|c|}{$\begin{array}{l}\text { Porcentagem de } \\
\text { emergência }\end{array}$} \\
\hline Tratamentos & Médias & Tratamentos & Médias & Tratamentos & Médias & Tratamentos & Médias \\
\hline NE $P_{1}$ & 68,21 & $\mathrm{NE} \mathrm{P}_{1}$ & 51,00 & NE $P_{1}$ & 61,68 & NE $P_{1}$ & 71,25 \\
\hline $\mathrm{NE} \mathbf{P}_{2}$ & 60,17 & E $P_{1}$ & 50,39 & $E P_{1}$ & 46,56 & NE $P_{2}$ & 61,61 \\
\hline E $P_{1}$ & $5,5,06$ & $\mathrm{NE} \mathrm{P}_{2}$ & 40,68 & $\mathrm{NE} \mathrm{P}_{2}$ & 46,28 & $E P_{1}$ & 60,52 \\
\hline $\mathrm{E} \boldsymbol{P}_{2}$ & 53,48 & $\mathrm{E} \mathrm{P}_{2}$ & 37,83 & $\mathrm{NEP}_{3}$ & 38,66 & $\mathrm{E} \mathrm{P}_{2}$ & 55,84 \\
\hline $\mathrm{NE}_{\mathbf{3}}$ & 51,57 & $\mathrm{NE}_{\mathbf{3}}$ & 37,42 & $\mathrm{E} \mathbf{P}_{\mathbf{2}}$ & 37,63 & $\mathrm{NE} \mathrm{P}_{3}$ & 45,64 \\
\hline $\mathrm{EP}_{\mathbf{3}}$ & 45,93 & $\mathrm{EP}_{\mathbf{3}}$ & 30,45 & $\mathrm{EP}_{3}$ & 32,53 & $\mathrm{EP}_{\mathbf{3}}$ & 38,34 \\
\hline $\begin{array}{l}\text { d.m.s. }-5 \% \\
\text { (Tukey) }\end{array}$ & 4,40 & & 5,24 & & 4,64 & & 4,04 \\
\hline C.V.\% & 6,57 & & 10,61 & & 8,80 & & 6,06 \\
\hline
\end{tabular}

De um modo geral, houve queda da germinação e do vigor de acordo com o aumento do periodo de armazenamento, para as sementes $\mathrm{NE}$. $\mathrm{O}$ vigor das submetidas ao tratamento $\mathrm{E}$, segundo os resultados da primeira contagem de germinação, da porcentagem de emergência e teste de cloreto de amônio decresceu significativamente com o decorrer do armazenamento; o mesmo não se constatou para a germinação, onde o decréscimo foi significativo apenas na terceira época $\left(\mathrm{P}_{3}\right)$.

Segundo os resultados de todos esses testes, as sementes do tratamento E, no terceiro teste $\left(\mathrm{E} \mathrm{P}_{3}\right)$, estavam significativamente mais deterioradas que as dos demais tratamentos.

O teste do cloreto de amônio revelou as diferenças entre os efeitos dos tratamentos, confirmando os resultados obtidos por ABDULAHI (1968), MOCKEL (1969), ABDULAHI \& VANDERLIP (1972), VANDERLIP e colaboradores (1973) e YAYOCK e outros (1975). Os resultados de porcentagem de emergência das plân tulas condizem com as observações de CAMARGO (1971), tendo acusado diferenças de vigor entre as sementes testadas.

Por outro lado, a ineficiência da primeira contagem de germinação, evidenciada por MOCKEL (1969), foi constatada no presente trabalho. Da mesma forma que o teste padrão, os resultados da primeira contagem de germinação revelaram apenas as diferenças muito acentuadas entre os tratamentos.

3. Interação Ambientes X Períodos de Armazenamento - As médias obtidas para esta interação achám-se no quadro 4. 


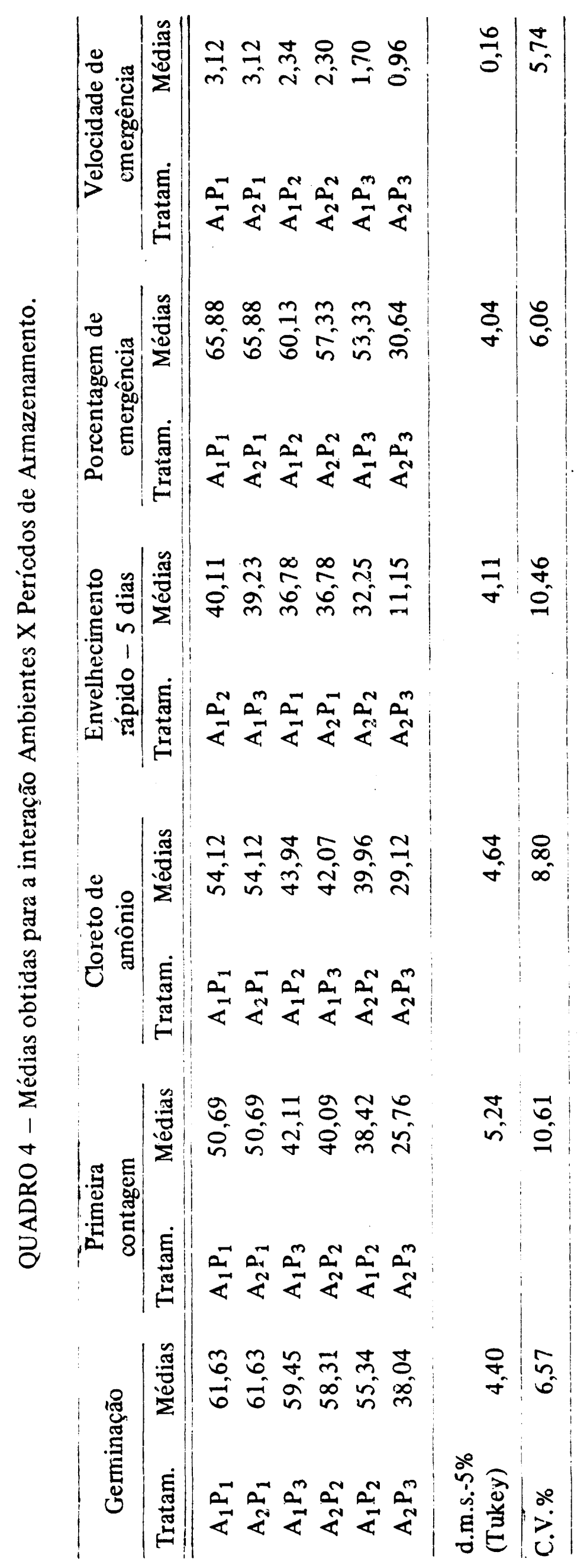


Observou-se, de um modo geral, efeito benéfico do armazenamento em ambiente controlado $\left(\mathrm{A}_{1}\right)$. As sementes armazenadas em ambiente normal, na terceira época $\left(\begin{array}{ll}A_{2} & P_{3}\end{array}\right)$ apresentaram-se mais deterioradas que as dos demais tratamentos. Segundo HARRINGTON (1972), a longevidade das sementes depende da viabilidade no início do período de armazenamento, mas é muito influenciada pelas condições de ambiente durante esse período.

4. Interação Envelhecimentos X Ambientes X Períodos de Armazenamento - Os valores médios observados para essa interação encontram-se no quadro 5.

QUADRO 5 - Médias obtidas para a interação Envelhecimentos X Ambientes X Períodos de Armazenamento.

\begin{tabular}{|c|c|c|c|}
\hline \multicolumn{2}{|c|}{ Velocidade de germinação } & \multicolumn{2}{|c|}{ Envelhecimento rápido -7 dias } \\
\hline Tratamentos & Médias & Tratamentos & Médias \\
\hline $\mathrm{NE} \mathrm{A}_{1} \mathrm{P}_{1}$ & 2,94 & $\mathrm{NE} \mathbf{A}_{1} \mathbf{P}_{3}$ & 36,83 \\
\hline $\mathrm{NE} \mathrm{A}_{2} \mathrm{P}_{1}$ & 2,94 & NE $A_{1} P_{1}$ & 32,76 \\
\hline $\mathrm{NE} \mathrm{A}_{2} \mathrm{P}_{2}$ & 2,87 & $\mathrm{NE} \mathrm{A}_{2} \mathrm{P}_{1}$ & 32,76 \\
\hline $\mathrm{E} \mathrm{A}_{2} \mathrm{P}_{2}$ & 2,79 & $E A_{1} P_{3}$ & 28,61 \\
\hline $\mathrm{NE} \mathbf{A}_{\mathbf{1}} \mathrm{P}_{\mathbf{3}}$ & 2,69 & $\mathrm{NE}_{\mathrm{A}_{1}} \mathrm{P}_{2}$ & 28,14 \\
\hline $\mathrm{NE} A_{1} P_{2}$ & $2,5 i$ & $\mathrm{E} \mathrm{A}_{1} \mathrm{P}_{2}$ & 21,40 \\
\hline$E A_{1} P_{1}$ & 2,52 & $E A_{1} P_{1}$ & 21,39 \\
\hline$E A_{2} P_{1}$ & 2,52 & $E A_{2} P_{1}$ & 21,39 \\
\hline$E A_{1} P_{3}$ & 2,46 & $\mathrm{NE} \mathrm{A}_{2} \mathrm{P}_{2}$ & 21,30 \\
\hline $\mathrm{E} \mathrm{A}_{\mathbf{2}} \mathbf{P}_{2}$ & 2,36 & $\mathrm{E} \mathrm{A}_{2} \mathrm{P}_{2}$ & 17,30 \\
\hline $\mathrm{NE} \mathrm{A}_{2} \mathrm{P}_{3}$ & 1,94 & $\mathrm{NE} \mathrm{A}_{2} \mathrm{P}_{3}$ & 9,27 \\
\hline$E A_{2} P_{3}$ & 1,70 & $\mathrm{E} \mathrm{A}_{2} \mathrm{P}_{3}$ & 8,13 \\
\hline $\begin{array}{l}\text { d.m.s. }-5 \% \\
\text { (Tukey) }\end{array}$ & 0,28 & & 4,12 \\
\hline C.V.\% & 5,61 & & 9,00 \\
\hline
\end{tabular}

Observou-se de um modo geral, efeitos significativos desfavoráveis do tratamento $E$ e favoráveis do armazenamento em câmara seca $\left(A_{1}\right)$ sobre a conservação de sementes.

As sementes armazenadas em ambiente normal, na terceira época ( $\mathrm{NE} \mathrm{A}_{2} \quad \mathrm{P}_{3}$ e $\left.\begin{array}{lll}\mathrm{A}_{2} & \mathrm{P}_{3}\end{array}\right)$, apresentaram-se significativamente mais deterioradas que as dos demais tratamentos. Por outro lado, as sementes do tratamento $E$ armazenadas em câmara seca $\left(E A_{1}\right)$ conservaram-se melhor que as do tratamento $\mathrm{NE}$ armazenadas em ambiente normal $\left(\mathrm{NE} \mathrm{A}_{2}\right)$, o que evidencia ainda mais o efeito benéfico do ambiente.

Resuitados semelhantes foram obtidos por MIRANDA (1967), que constatou prejuízos causados pela fiutuação da umidade relativa ambiente, durante o período de armazenamento, sobre a qualidade de sementes de sorgo. No presente trabalho verificou-se que a viabilidade inicial das sementes e as condições de ambiente influenciaram a germinação e o vigor, mas os efeitos do ambiente mostraram-se mais significativos. 
$\mathrm{O}$ índice de velocidade de germinação mostrou-se eficiente para avaliar o vigor das sementes, o mesmo ocorrendo com o envelhecimento rápido. Porém, o período de 7 dias revelou-se muito drástico para as sementes, chegando ao ponto de praticamente impedir a germinação; o mesmo se verificou com o período de 5 dias, na terceira época.

5. Correlação entre os Métodos - No presente trabalho, as correlaçốes entre os testes de germinação e de vigor foi considerada significativa quando os coeficientes de determinação $\left(\mathrm{r}^{2}\right)$ excederam o valor 0,49 ; nestes casos, há mais de $49 \%$ de explicação da variação pela correlação.

Os valores dos coeficientes de correlação simples encontrados para todas as combinações entre os métodos encontram-se nos quadros 6, 7, 8 e 9

QUADRO 6 - Coeficientes de correlação simples (r) entre testes de germinação e de vigor com sementes não envelhecidas armazenadas em câmara seca $\left(\mathrm{NE} \mathrm{A}_{1}\right)$.

\begin{tabular}{|c|c|c|c|c|c|c|c|c|}
\hline & 1 & 2 & 3 & 4 & 5 & 6 & 7 & 8 \\
\hline 1. Germinação & 1,00 & $-0,682$ & $-0,569$ & $-0,286$ & 0,545 & $-0,488$ & $-0,298$ & $-0,305$ \\
\hline 2. Primeira & & & & & & & & \\
\hline Contagem & $-0,682$ & 1,00 & 0,557 & 0,410 & $-0,409$ & 0,474 & 0,230 & 0,267 \\
\hline $\begin{array}{l}\text { 3. Velocidade de } \\
\text { Germinação }\end{array}$ & $-0,569$ & 0,557 & 1,00 & 0,608 & $-0,688$ & 0,099 & 0,501 & 0,586 \\
\hline $\begin{array}{l}\text { 4. Cloreto de } \\
\text { Amônio }\end{array}$ & $-0,286$ & 0,410 & 0,608 & 1,00 & $-0,442$ & $-0,127$ & $0,712 * *$ & $0,830^{* *}$ \\
\hline $\begin{array}{l}\text { 5. Envelhecimento } \\
\text { rápido ( } 5 \text { dias) }\end{array}$ & 0,545 & $-0,409$ & $-0,688$ & $-0,442$ & 1,00 & $-0,054$ & $-0,347$ & $-0,480$ \\
\hline $\begin{array}{l}\text { 6. Envelhecimento } \\
\text { rápido ( } 7 \text { dias) }\end{array}$ & $-0,488$ & 0,474 & 0,099 & $-0,127$ & $-0,054$ & 1,00 & $-0,310$ & $-0,310$ \\
\hline $\begin{array}{l}\text { 7. Porcentagem de } \\
\text { emergência }\end{array}$ & $-0,298$ & 0,230 & 0,501 & $0,712 * *$ & $-0,347$ & $-0,310$ & 1,00 & $0,927 * *$ \\
\hline $\begin{array}{l}\text { 8. Velocidade de } \\
\text { emergência }\end{array}$ & $-0,305$ & 0,267 & 0,586 & $0,830^{* *}$ & $-0,480$ & $-0,310$ & $0,927^{* *}$ & 1,00 \\
\hline
\end{tabular}


QUADRO 7 - Coeficientes de correlaçẩo simples (r) ertre testes de germinação $\epsilon$ de vigor com sementes envelhecidas armazenadas em câmara seca $\left(E A_{1}\right)$.

\begin{tabular}{|c|c|c|c|c|c|c|c|c|}
\hline 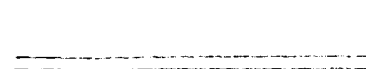 & 1 & 2 & 3 & 4 & 5 & 6 & 7 & 8 \\
\hline 1. Germinação & 1,00 & $-0,161$ & $-0,385$ & $-0,136$ & 0,084 & 0,516 & $-0,298$ & $-0,327$ \\
\hline $\begin{array}{l}\text { 2. Primeira } \\
\text { Contagem }\end{array}$ & $-0,161$ & 1,00 & $-0,178$ & 0,799 ** & 0,087 & $-0,526$ & 0,496 & 0,664 \\
\hline $\begin{array}{l}\text { 3. Velocidade de } \\
\text { Germinação }\end{array}$ & $-0,385$ & $-0,178$ & 1,00 & $-0,109$ & 0,177 & $-0,302$ & 0,336 & 0,205 \\
\hline $\begin{array}{l}\text { 4. Cloreto de } \\
\text { Amônio }\end{array}$ & $-0,136$ & $0,799 * *$ & $-0,109$ & 1,00 & $-0,009$ & $-0,483$ & 0,741 ** & $0,701 *$ \\
\hline $\begin{array}{l}\text { 5. Envelhecimento } \\
\text { rápido (5 dias) }\end{array}$ & 0,084 & 0,087 & 0,177 & $-0,009$ & 1,00 & $-0,157$ & 0,008 & $-0,079$ \\
\hline $\begin{array}{l}\text { 6. Envelhecimento } \\
\text { rápido ( } 7 \text { dias) }\end{array}$ & 0,516 & $-0,526$ & $-0,302$ & $-0,483$ & $-0,157$ & 1,00 & $-0,722 * *$ & $-0,766$ ** \\
\hline $\begin{array}{l}\text { 7. Porcentagem de } \\
\text { emergếncia }\end{array}$ & $-0,298$ & 0,496 & 0,336 & $0,741^{* *}$ & 0,008 & $-0,722^{* *}$ & 1,00 & 0,881 ** \\
\hline $\begin{array}{l}\text { 8. Velocidade dè } \\
\text { emergência }\end{array}$ & $-0,327$ & 0,664 & 0,205 & 0,701 ** & $-0,079$ & $-0,766 * *$ & $0,881 * *$ & 1,00 \\
\hline
\end{tabular}

QUADRO 8 - Coeficientes de correlação simples (r) entre testes de germinação e de vigor com sementes não envelhecidas armazenadas em ambiente normal $\left(\mathrm{NE} \mathrm{A}_{2}\right)$.

\begin{tabular}{|c|c|c|c|c|c|c|c|c|}
\hline & 1. & 2 & 3 & 4 & 5 & 6 & 7 & 8 \\
\hline 1. Germinação & 1,00 & $0,882 * *$ & 0,921 ** & $0,858 * *$ & $0,937^{* *}$ & $0,893 * *$ & $0,962 * *$ & $0,948^{* *}$ \\
\hline $\begin{array}{l}\text { Primeira } \\
\text { Contagem }\end{array}$ & $0,882^{* *}$ & 1,00 & $0,856^{* *}$ & 0,913 ** & $0,856 * *$ & $0,951 * *$ & 0,901 ** & 0,937 \\
\hline $\begin{array}{l}\text { 3. Velocidade de } \\
\text { Germinação }\end{array}$ & $0,921^{\text {*** }}$ & $0,856^{* *}$ & 1,00 & $0,789 * *$ & $0,931^{* *}$ & $0,867^{* *}$ & $0,916^{* *}$ & $0,9099_{1 .}^{* *}$ \\
\hline $\begin{array}{l}\text { 4. Cloreto de } \\
\text { Amônio }\end{array}$ & $0,858^{* *}$ & $0,913^{* *}$ & $0,789 * *$ & 1,00 & $0,828^{* *}$ & $0,936 * *$ & $0,882^{* *}$ & 0,929 * \\
\hline $\begin{array}{l}\text { 5. Envelhecimento } \\
\text { rápido ( } 5 \text { dias) }\end{array}$ & $0,937^{* *}$ & 0,856 ** & $0,931 * *$ & 0,828 ** & 1,00 & 0,890 ** & $0,953^{* *}$ & 0,935 ** \\
\hline $\begin{array}{l}\text { 6. Envelhecimento } \\
\text { rápido ( } 7 \text { dias) }\end{array}$ & $0,893 * *$ & 0,951 ** & $0,867 * *$ & $0,936^{* *}$ & $0,890 * *$ & 1,00 & $0,926^{* *}$ & $0,965^{* *}$ \\
\hline $\begin{array}{l}\text { 7. Porcentagem de } \\
\text { emergência }\end{array}$ & $0,962^{* *}$ & $0,901 * *$ & 0,916 ** & $0,882 * *$ & $0,953 * *$ & $0,926^{* *}$ & 1,00 & $0,978 * *$ \\
\hline $\begin{array}{l}\text { 8. Velocidade de } \\
\text { emergência }\end{array}$ & 0,948 ** & 0,937 ** & $0,909 * *$ & $0,929 * *$ & 0,935 ** & $0,965 * *$ & 0,978 ** & 1,00 \\
\hline
\end{tabular}


QUADRO 9 - Coeficientes de correiação simples ( $r$ ) entre testes de germinação e de vigor com sementes envelhecidas armazenadas em ambiente normal $\left(E A_{2}\right)$.

\begin{tabular}{|c|c|c|c|c|c|c|c|c|}
\hline & 1 & 2 & 3 & 4 & 5 & 6 & 7 & 8 \\
\hline 1. Germinação & 1,00 & $0,857^{* *}$ & 0,867 ** & $0,813 * *$ & $0,946 * *$ & $0,887 * *$ & $0,921 * *$ & $0,873^{* *}$ \\
\hline 2. Primeira & & & & & & & & \\
\hline Contagem & 0,857 ** & 1,00 & $0,879 * *$ & $0,884 * *$ & $0,888 * *$ & $0,910 * *$ & $0,933 * *$ & $0,956 * *$ \\
\hline 3. Velocidade de & & & & & & & & \\
\hline Germinação & $0,867^{* *}$ & $0,879 * *$ & 1,00 & $0,800^{* *}$ & $0,846^{* *}$ & $0,883 * *$ & $0,938 * *$ & $0,920 * *$ \\
\hline 4. Cloreto de & & & & & & & & \\
\hline Amônio & $0,813^{* *}$ & $0,884 * *$ & $0,800 *$ & 1,00 & $0,818 * *$ & $0,865 * *$ & 0,875 ** & $0,928 * *$ \\
\hline $\begin{array}{l}\text { 5. Envelhecimento } \\
\text { rápido ( } 5 \text { dias) }\end{array}$ & $0,946 * *$ & 0,888 ** & 0,846 ** & $0,818^{* *}$ & 1,00 & $0,904 * *$ & $0,916 * *$ & $0,899 * *$ \\
\hline $\begin{array}{l}\text { 6. Envelhecimento } \\
\text {-rápido ( } 7 \text { dias) }\end{array}$ & $0,887^{* *}$ & $0,910^{* *}$ & $0,883 * *$ & $0,865^{* *}$ & $0,904^{* *}$ & 1,00 & $0,924 * *$ & $0,944^{* *}$ \\
\hline $\begin{array}{l}\text { 7. Porcentagem de } \\
\text { emergência }\end{array}$ & 0,921 ** & $0,933 * *$ & $0,938^{* *}$ & $0,875^{* *}$ & $0,916 * *$ & $0,924 * *$ & 1,00 & 0,971 ** \\
\hline $\begin{array}{l}\text { 8. Velocidade de } \\
\text { emergência }\end{array}$ & $0,873 * *$ & $0,956^{* *}$ & $0,920^{* *}$ & $0,928 * *$ & $0,899^{* *}$ & $0,944 * *$ & $0,971^{* *}$ & 1,00 \\
\hline
\end{tabular}

Os resultados do teste do cloreto de amônio, de velocidade e de porcentagem de emergência correlacionaram-se positivamente entre si, tanto para as sementes envelhecidas como as não envelhecidas armazenadas em câmara seca (E $\boldsymbol{\Lambda}_{1}$ e $N E A_{1}$ ). Altos coeficientes de correlação entre cloreto de amônio e porcentagem de èmergência no campo foram também obtidos por ABDULAHI (1968), MOCKEL (1969), ABDULAHF \& VAN. DERLIP (1972), VANDERLIP e colaboradores (1973) e YAYOCK e outrós (1975).

Os altos indices de correlação entre velocidade e porcentagem de emergência das plântulas sugerem que talvez não se justifique a utilização conjunta dos dois métodos; a avaliação diária da emergência das plântulas é mas trabalhosa e exige a adoção de critérios minuciosos, dificilmente padronizáveis.

Houve correlação negativa entre envelhecimento rápido ( 7 dias) e a velocidade e porcentagem de emergência das plântulas, conforme se verifica através do exame do quadro 7. Esta variação foi considerada anormal e, provavelmente, causada por erro acidental.

Por outro lado, as altas correlações observadas nos quadros 8 e 9 demonstraram que, quando as sementes testadas eram de qualidade inferior, todos os métodos foram eficientes.

\section{CONCLUSO̊ES}

As análises dos dados e a interpretação dos resultados do presente trabalho permitiram concluir que a eficiência dos diferentes métodos estudados deperide da qualidade das sementes testadas. 
O teste padrão e a primeira contagem de germinação revelaram apenas as diferenças marcantes entre os tratamentos. Dos métodos de laboratório, a velocidade de germinação e cloreto de amônio foram os mais eficientes para a avaliação do vigor de sementes.

Por outro lado, os períodos de cinco e de sete dias empregados no teste de envelhecimento rápido foram muito drásticos, o que sugere a necessidade de novos estudos para a aplicação deste método para sementes de sorgo.

\section{SUMMARY}

\section{COMPARISON OF VIGOR TESTS FOR EVALUATING SORGHUM SEED QUALITY}

Six methods were tested comparatively for the evaluation of sorghum seed quality in an attempt to determine the efficiency of the different methods.

The following methods were tested: standard germination, first count, speed of germination, ammonium chloride test, accelerated aging and seedling emergence.

Results showed that among the tests performed, standard germination and first count were the least sensitive in the evaluation of the physiological condition of seeds. The ammonium chloride and speed of germination tests diferenciated more accurately the degree of deterioration of seeds.

\section{LITERATURA CITADA}

ABDULAHI, A., 1968. Seed vigor measurements and their use in predicting field establishment of grain sorghum. M.S. Thesis, Kansas Sta. Univ., 60 p.

ABDULAHI, A. \& VANDERLIP, R.L., 1972. Relationship of vigor tests and seed source and size to sorghum seedling establishment. Agron. J., 64(2):143-144.

BYRD, H.W. \& DELOUCHE, J.C., 1972. Deterioration of soybean seed in storage. Proc. Assoc. Off. Seed Anal., $61: 41-48$.

CAMARGO, C.P., 1971. Effect of seed vigor upon seed performance and yield of grain sorghum (Sorghum bicolor L., Moench). M.S. Thesis, Miss. Sta. Univ., 61 p.

CLARK, B.E., 1953. Relationship between certain laboratory tests and the field germination of sweet corn. Proc. Assoc. Off. Seed Anal., $43: 42-44$.

DELOUCHE, J.C., 1963. Seed deterioration. Seed World, 92(4):14-15.

DELOUCHE, J.C. \& BASKIN, C.C., 1973. Accelerated aging techniques for predicting the relative storability of seed lots. Seed Sci. and Technol., $1.427-452$.

GRABE, D.F., 1965. Agronomic significance of seed deterioration. Agr. Abstr., $57: 40$.

HARRINGTON, J.F., 1972. Seed storage and longevity; in Seed Biology. Academic Press, New York, vol. III. 422 p. 
HELMER, J.D., 1967. Predicting seed storability. Proc. Short Course for Seedsmen, Miss. Sta. Univ., $65: 68$.

MINISTÉRIO DA AǴRICULTUURA, 1967. Regras para a Análise de Sementes. Escritório da Produção Vegetal. Equipe Técnica de Sementes e Mudas. 120 p.

MirandA, P., 1967. Conservação de sementes de sorgo. Bol. Téq. IPA, Pernambuco, 21 27-33.

MOCKEL, F.E., 1969. Relationship of seed vigor among grain sorghum hybrids and field establishment. M.S. Thesis, Kansas Sta. Univ., 46 p.

SWANSON, A.F. \& HUNTER, R., 1936. Effect of germination and seed size on sorghum stands. J. Amer. Soc. Agron., 28:997-1004.

VANDERLIP, R.L., MOCKEL, F.E. \& JAN, H., 1973. Evaluation of vigor tests for sorghum. Agron. J., 65(3):486-488.

YAYOCK, J.Y., JAN, H. \& VANDERLIP, R.L., 1975. Temperature, time and $\mathrm{NH}_{4} \mathrm{Cl}$ concentration in vigor testing of sorghum seed. Agron. J., 67(2):241-242. 
